\title{
Risk factors of perfusion and diffusion abnormalities on MRI in hemispheric TIA: a case-control study
}

\author{
Yue Wang ${ }^{1,2,3}$, Jingjing Xiao $^{1}$, Yu Luo ${ }^{4}$, Shaoshi Wang ${ }^{1}$, Huazheng Liang ${ }^{1,3}$, Lingjing Jin ${ }^{2}$ \\ ${ }^{1}$ Department of Neurology, Shanghai Fourth People's Hospital affiliated to Tongji University School of Medicine, Shanghai 200081, China; \\ ${ }^{2}$ Department of Neurology, Shanghai Tongji Hospital, Tongji University, Shanghai 200065, China; ${ }^{3}$ Department of Neurology, Translational \\ Research Institute of Brain and Brain-like Intelligence, ${ }^{4}$ Department of Radiology, Shanghai Fourth People's Hospital Affiliated to Tongji University \\ School of Medicine, Shanghai 200081, China \\ Contributions: (I) Conception and design: Y Wang, H Liang, L Jin; (II) Administrative support: None; (III) Provision of study materials or patients: \\ None; (IV) Collection and assembly of data: Y Wang, H Liang, L Jin, Y Luo, J Xiao; (V) Data analysis and interpretation: Y Wang, H Liang, L Jin, \\ S Wang; (VI) Manuscript writing: All authors; (VII) Final approval of manuscript: All authors. \\ Correspondence to: Lingjing Jin, MBBS, PhD. Department of Neurology, Shanghai Tongji Hospital, Tongji University, No. 389 Xincun Road, \\ Shanghai 200065, China. Email: lingjingjin@163.com; Huazheng Liang, MBBS, PhD. Department of Neurology, Shanghai Fourth People’s Hospital \\ affiliated to Tongji University School of Medicine, 1878 North Sichuan Road, Shanghai 200081, China. Email: andyliang2018@126.com.
}

Background: To assess the prevalence and potential predictors of MR diffusion and perfusion abnormalities in a Chinese population with hemispheric transient ischemic attacks (TIA).

Methods: Patients with temporary (<24 hours) focal cerebral dysfunction of probable vascular origin were considered to be potential candidates for this study in the emergency room. Those who were admitted to the stroke center of Shanghai Fourth People's Hospital affiliated to Tongji University between January 2015 and December 2018 were recruited to the present study. MRI, including both diffusion-weighted imaging (DWI) and perfusion-weighted imaging (PWI), was performed within 7 days after the last symptom attack. Time to maximum of the residue function (Tmax) maps were auto-evaluated using the RAPID software to determine hypoperfusion. Multivariate analysis was used to assess the correlation of MR findings with clinical variables, medical history, cardio-metabolic characteristics, and the ABCD2 scores (age, blood pressure, clinical features, symptom duration and diabetes).

Results: Sixty-six out of 207 patients met the inclusion criteria. Baseline MRI showed DWI lesions in 20 patients (30.3\%). The prevalence of MR perfusion Tmax $>4 \mathrm{~s}>0 \mathrm{~mL}$ and $\geq 10 \mathrm{~mL}$ were $77.3 \%(51 / 66)$ and 50\% (33/66), respectively. Male patients tended to develop DWI lesions after a TIA. Limb weakness was an independent factor associated with MR perfusion abnormalities ( $T$ max $>4 \mathrm{~s} \geq 10 \mathrm{~mL}$ ) in this Chinese population (adjusted $\mathrm{OR}=7.41,95 \% \mathrm{CI}: 1.57-34.89, \mathrm{P}=0.011$ ).

Conclusions: Our results suggest that limb weakness is a strong predictor of perfusion abnormalities calculated by RAPID on Tmax maps of hemispheric TIA patients without DWI positive findings. Male patients are more likely to develop cerebral infarction.

Keywords: Magnetic resonance imaging (MRI); diffusion-weighted imaging; perfusion weighted imaging; transient ischemic attack; risk factors; correlation analysis

Submitted Jun 25, 2019. Accepted for publication Nov 15, 2019.

doi: $10.21037 /$ atm.2019.12.69

View this article at: http://dx.doi.org/10.21037/atm.2019.12.69

(C) Annals of Translational Medicine. All rights reserved. 


\section{Introduction}

Transient ischemic attack (TIA) is redefined as a transient episode of neurological symptoms attributable to dysfunction of focal brain areas without evidence of acute ischemic infarction (1). However, if MRI is not available immediately, the time-based TIA definition with symptoms lasting less than 24 hours was still used in China (1). The population of TIA survivors at any given time in China is as large as 10 to 12 million according to a community-based multicenter study (2). TIAs are associated with future recurrent ischemic attacks. An accurate diagnosis of the cause is essential to ensure appropriate management is applied and to dramatically reduce the risk of developing recurrent ischemic events (3-5).

Diagnosing the ischemic mechanism of transient neurologic dysfunction is challenging, even for experienced neurologists $(6,7)$. In recent studies, diffusion-weighted imaging (DWI) showed acute ischemic lesions among $30-40 \%$ of patients referred for a clinically defined TIA (8-11). High-resolution DWI (3T, $2.5 \mathrm{~mm}$ without gap slice thickness) can detect lesions in more than half of these patients with TIA (12). DWI lesions are associated with recurrent ischemic attacks after a TIA $(9,13)$. However, patients without DWI lesions remain a source of uncertainty and their management is challenging. Although many of these subjects have had transient cerebral ischemia, the extent of hemodynamic damage does not reach the threshold of tissue injury. Perfusion weighted imaging (PWI) demonstrates acute ischemic lesions among $20-40 \%$ of TIA patients who have normal DWI $(8,9,14-16)$. Furthermore, PWI abnormalities are associated with recurrent TIAs, strokes, and persistent deterioration of neurologic symptoms $(9,15,17,18)$.

Cerebral perfusion can be visualized by intravenously injecting clinically approved gadolinium-containing contrast agents $(19,20)$. The size of hypoperfusion was estimated from the volume of tissue where delayed arrival of an injected contrast agent [the time when the residual function reaches its maximum (Tmax)] exceeding 6 seconds was displayed (21-23). Tmax maps can reveal ischemic abnormalities in TIA patients with negative DWI findings (24). Tmax $>4$ s seems to be optimal for early assessment of hypoperfusion in TIA patients (25). However, little research has been conducted in Chinese populations. The purpose of the present study, therefore, was to assess the prevalence and potential predictors of MR diffusion and perfusion abnormalities in a Chinese population with TIAs.

\section{Methods}

\section{Subjects}

Patients with a temporary ( $<24$ hours) focal cerebral dysfunction of probable vascular origin were considered as potential candidates for this study at emergency room. Some of these subjects were admitted to the comprehensive Stroke Center of Shanghai Fourth People's Hospital affiliated to Tongji University with a discharge diagnosis of TIA (DWI negative) or minor stroke (DWI positive) between January 2015 and December 2018. These subjects were recruited to the present study.

The inclusion criteria for this study: (I) patients presented with a TIA and evaluated by two certified stroke neurologists at the time of admission and discharge; (II) MR imaging including both DWI and PWI available at the time of hospitalization; (III) The time when the residue function reaches its maximum (Tmax) maps were assessed using the RAPID software (iSchemaView USA, Version 4.9) (23), independently.

The exclusion criteria: (I) patients with TIA did not have baseline perfusion or their diffusion status was not assessed; (II) patients received revascularization therapy (thrombolysis/thrombectomy).

Radiologists blinded to clinical information independently evaluated the presence of acute ischemic lesions detected on DWI/PWI. Demographic data, clinical variables, risk factors, neurologic deficits, $\mathrm{ABCD}^{2}$ scores, frequency of attacks, lasting time of TIA, time between MRI scan and onset were documented for each patient.

\section{Image acquisition}

MRI scans were performed on a 1.5-T Avanto scanner (Siemens, Erlangen, Germany). The imaging protocol for this study included DWI, PWI, FLAIR and time-of-flight MR angiography (TOF-MRA). Imaging parameters were listed as below. The head coil is an-8-channel phased-array coil. DWI: 19 slices, $192 \times 192$ matrices; slice thickness = $5.5 \mathrm{~mm}$; TR/TE, $3,600 / 102 \mathrm{~ms}$; field of view $=230 \mathrm{~mm}^{2}$, $\mathrm{b}=0$ and $1,000 \mathrm{~s} / \mathrm{mm}^{2}$; EPI factor $=192$; bandwidth $=$ $964 \mathrm{~Hz} /$ pixel. FLAIR: 18 slices, 256×190 matrices; slice thickness $=5.5 \mathrm{~mm}$; TR/TE, 4,000/92 ms; field of view = $230 \mathrm{~mm}^{2}$; TI $=1,532.6 \mathrm{~ms}$; bandwidth $=190 \mathrm{~Hz} / \mathrm{Px}$; flip 


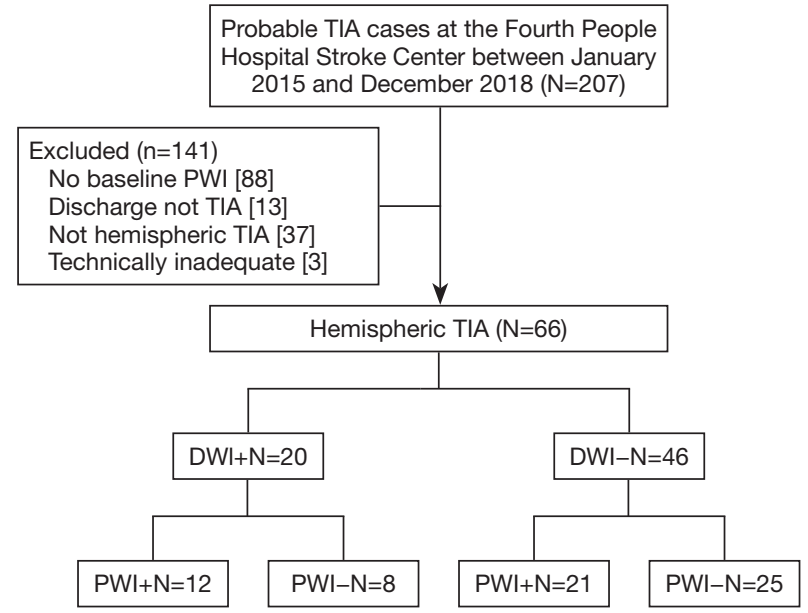

Figure 1 Flowchart of patient recruitment. DWI, diffusionweighted imaging; TIA, transient ischemic attack; PWI, perfusion weighted imaging.

angle $=150^{\circ}$. Dynamic susceptibility contrast PWI (DSCPWI): 19 slices, $128 \times 128$ matrices; slice thickness $=5 \mathrm{~mm}$; TR/TE, $1,590 / 32 \mathrm{~ms}$; measurements $=50$; field of view $=230 \mathrm{~mm}^{2}$; band width $=1,346 \mathrm{~Hz} /$ pixel; flip angle $=90^{\circ}$. A Gd-DTPA contrast agent (gadopentetate dimeglumine injection; Shanghai Pharmaceutical Corporation, Shanghai, China) was injected intravenously $(0.2 \mathrm{mmol} / \mathrm{kg}$ body weight) at a rate of $4 \mathrm{~mL} / \mathrm{s}$ after a bolus with $30 \mathrm{~mL}$ saline. Three-dimensional time-of-flight MRA of the internal carotid artery (ICA) and intracranial circulation: $241 \times 256$ matrices, slice thickness $=0.7 \mathrm{~mm}$; TR/TE, $25 / 7 \mathrm{~ms}$; field of view $=180 \mathrm{~mm}^{2}$; Bandwidth $=100 \mathrm{~Hz} / \mathrm{PX}$; flip angle $=25^{\circ}$.

\section{Post-processing}

Estimates of hypoperfusion on PWI were calculated using the RAPID software (iSchemaView USA, Version 4.9), which is an automated imaging post-processing system $(23,26)$. Volumes of Tmax delay $>4$ seconds were used to determine hypoperfusion deficits in TIA patients with a negative DWI $(9,15)$.

Arterial input functions from the proximal middle cerebral artery in both hemispheres were semi-automatically identified and used to generate perfusion maps (27).

\section{Ethics}

Ethical approval for this study was obtained from the Human Research Ethics Committee of Shanghai Fourth
People's Hospital Affiliated to Tongji University School of Medicine. Written informed consent was obtained from all subjects.

\section{Statistical analysis}

Continuous parameters were presented as mean \pm standard deviation (SD) or median with interquartile range (IQR); categorical variables were summarized as independent proportions. Baseline information of patients with or without MRI diffusion abnormalities was compared using $t$-test or Mann-Whitney $\mathrm{U}$ test for continuous variables and $\chi^{2}$ or Fisher's exact test for categorical variables. We used logistic regression analysis to identify independent predictors of DWI-positive and PWI-positive TIA, respectively. Multivariate regression modelling was used to correlate DWI/PWI with related risk factors with $\mathrm{P}$ values $<0.20$. All correlation data were presented as odds ratios (OR) with their corresponding 95\% confidence intervals (CI) and $\mathrm{P}$ values. Statistical significance was considered when $\mathrm{P}<0.05$. Data analysis was performed using IBM SPSS (version 20.0) for Windows (SPSS Inc., Chicago, IL, USA).

\section{Results}

In the present study, 207 patient records were evaluated for suspected TIA, among which, 66 (27 women, 39 men; age range: $40-86$ years; mean, 70 years) had technically adequate DWI and PWI scans. Eighty-eight patients were excluded because their baseline PWI was not available after their TIA, 13 patients were excluded because they were not given a discharge diagnosis of a TIA, 37 patients were excluded due to non-hemispheric TIAs and 3 had technically inadequate MR scans (Figure 1).

\section{Baseline characteristics}

Baseline characteristics of patients included in the present study were shown in Table 1 and Table S1. A total of 66 subjects, including 39 males and 27 females, were included in the present study. The average age of these patients was $70 \pm$ 11 years old and their median (IQR) $\mathrm{ABCD}^{2}$ score was 4 [35]. Baseline perfusion scans were evaluated after a median (IQR) delay of 5 [2-11] days from the first symptom onset or 5 (IQR 2-7) days from the last symptom attack. Their median (IQR) symptom duration was 20 [5-68] minutes and the median times of TIAs at baseline were 1 (IQR 1-3). The median (IQR) Tmax $>4$ s volume was 9 [3-67]. The 
Table 1 Comparison of baseline demographic and clinical characteristics between TIA patients ( $\mathrm{n}=66)$ with and without DWI lesions

\begin{tabular}{|c|c|c|c|c|c|}
\hline Characteristics & Total $(n=66)$ & $\mathrm{DWI}+(\mathrm{n}=20)$ & DWI- $(n=46)$ & $z, t$, or $\chi^{2}$ & $P$ value \\
\hline Male, n (\%) & $39(59.1)$ & $15(75.0)$ & $24(52.2)$ & 3.004 & 0.083 \\
\hline \multicolumn{6}{|l|}{ Medical history, n (\%) } \\
\hline Hypertension & $41(62.1)$ & $13(65.0)$ & $28(60.9)$ & 0.101 & 0.751 \\
\hline Atrial fibrillation & $5(7.6)$ & $3(15.0)$ & $2(4.3)$ & 2.225 & 0.136 \\
\hline Smoking & $18(27.3)$ & $8(40.0)$ & $10(21.7)$ & 2.343 & 0.126 \\
\hline Drinking & $10(15.2)$ & $4(20.0)$ & $6(13.0)$ & 0.525 & 0.476 \\
\hline Prior TIA or stroke & $21(31.8)$ & $5(25.0)$ & $16(34.8)$ & 0.615 & 0.433 \\
\hline $2 \mathrm{hBG}$ & $8.2(6.3-11.5)$ & $9.1(6.1-11.5)$ & $7.8(6.3-11.5)$ & 0.460 & 0.654 \\
\hline Triglyceride & $1.4(1.1-2.3)$ & $1.3(1.1-1.8)$ & $1.7(1.1-2.5)$ & 1.179 & 0.238 \\
\hline Total cholesterol & 4.41 .1 & $4.4 \pm 0.8$ & $4.4 \pm 1.3$ & 0.169 & 0.866 \\
\hline LDL & $2.2(1.8-2.9)$ & $2.7(1.9-2.9)$ & $2.1(1.6-2.8)$ & 1.221 & 0.222 \\
\hline Hcy & $12(10.4-15.6)$ & $11.9(10.5-15.7)$ & $12(10.3-15.6)$ & 0.174 & 0.862 \\
\hline \multicolumn{6}{|l|}{ Symptoms, n (\%) } \\
\hline Weakness & $24(36.4)$ & $6(30.0)$ & $18(39.1)$ & 0.502 & 0.479 \\
\hline Speech impairment & $32(48.5)$ & $9(45.0)$ & $23(50.0)$ & 0.140 & 0.709 \\
\hline Days_inhos, d, median (IQR) & $11(9-13.3)$ & $12(9-14)$ & $10(8.8-13)$ & 1.165 & 0.244 \\
\hline Perfusion_first, d, median (IQR) & $5.2(2-11.3)$ & $6.6(4.3-15.5)$ & $5(1.8-10.6)$ & 1.605 & 0.108 \\
\hline Perfusion_last, d, median (IQR) & $5(2-6.8)$ & $5.2(3.2-7.1)$ & $4.3(1.2-6.2)$ & 1.361 & 0.173 \\
\hline Stenosis (50\%), n (\%) & $17(25.8)$ & $6(30.0)$ & $11(23.9)$ & 0.270 & 0.603 \\
\hline Tmax >4 s, median (IQR) & $9(3-67)$ & $23(3.3-101)$ & $8.5(0-41.5)$ & 1.053 & 0.292 \\
\hline
\end{tabular}

IQR, interquartile range; Tmax $>4 \mathrm{~s}$, time to maximum of the residue function $\geq 4$ seconds; FBG, fasting blood-glucose; LDL, low-density lipoprotein cholesterol; ESR, erythrocyte sedimentation rate; SBP, systolic blood pressure; Days_inhos, days in hospital; Days_before, days from onset to hospitalization; Times_before, times from onset to hospitalization; Perfusion_first, symptom onset to the first MR perfusion (days); $A B C D^{2}$ score = age 60 (1 point), SBP 140 or DBP 90 mmHg (1 point), clinical features as unilateral weakness (2 points) or speech impairment without weakness (1 point), symptom duration 60 min (2 points) or 10-59 min (1 point), diabetes (1 point). Significant difference at $\alpha<0.05$. TIA, transient ischemic attack; DWI, diffusion-weighted imaging. 
median (IQR) fasting blood-glucose (FBG) was 5.4 (4.96.0) $\mathrm{mmol} / \mathrm{L}$. A history of hypertension was present in $62.1 \%(41 / 66)$ of patients, diabetes mellitus in $24.2 \%$ $(16 / 66)$, atrial fibrillation in $7.6 \%(5 / 66)$, history of strokes in $31.8 \%$ (21/66), smoking in $27.3 \%(18 / 66)$, and stenosis $(50 \%)$ in $25.8(17 / 66)$ of patients. Paresis and speech impairment were present in $36.4 \%(24 / 66)$ and $48.5 \%$ (32/66) of patients, respectively (Table 1).

Baseline MRI showed DWI lesions in 20 patients

Table 2 Summary of DWI and PWI findings

\begin{tabular}{lccc}
\hline & DWI+ & DWI- & Total \\
\hline PWI+ & $12(18.18)$ & $21(31.81)$ & $33[50]$ \\
PWI- & $8(12.12)$ & $25(37.87)$ & $33[50]$ \\
Total & $20(30.3)$ & $46(69.69)$ & \\
\hline
\end{tabular}

DWI, diffusion-weighted imaging; PWI, perfusion weighted imaging.
(30.3\%), and PWI lesions in $33(50 \%)$ patients (Tmax $>4 \mathrm{~s}$ $\geq 10 \mathrm{~mL}$ ); among the PWI-positive patients, 21 (31.8\%) did not have an acute DWI lesion (Table 2). Figure $2 A, B$ demonstrated a case with a positive DWI and a positive PWI, while Figure $2 C, D$ showed a case with a positive PWI and a negative DWI.

MRA scans with adequate quality of both the carotid and intracranial vessels were available in the present study. MRA stenosis (greater than 50\%) was detected in 17 (25.8\%) patients. Among the 46 patients without DWI lesions, $11(23.9 \%)$ had an ipsilateral arterial stenosis. Among the 20 cases with a DWI lesion, $6(30 \%)$ had an ipsilateral arterial stenosis.

\section{Comparison of demographic and clinical characteristics among TIA patients with and without a MR diffusion lesion}

Table 1 presented the demographic characteristics
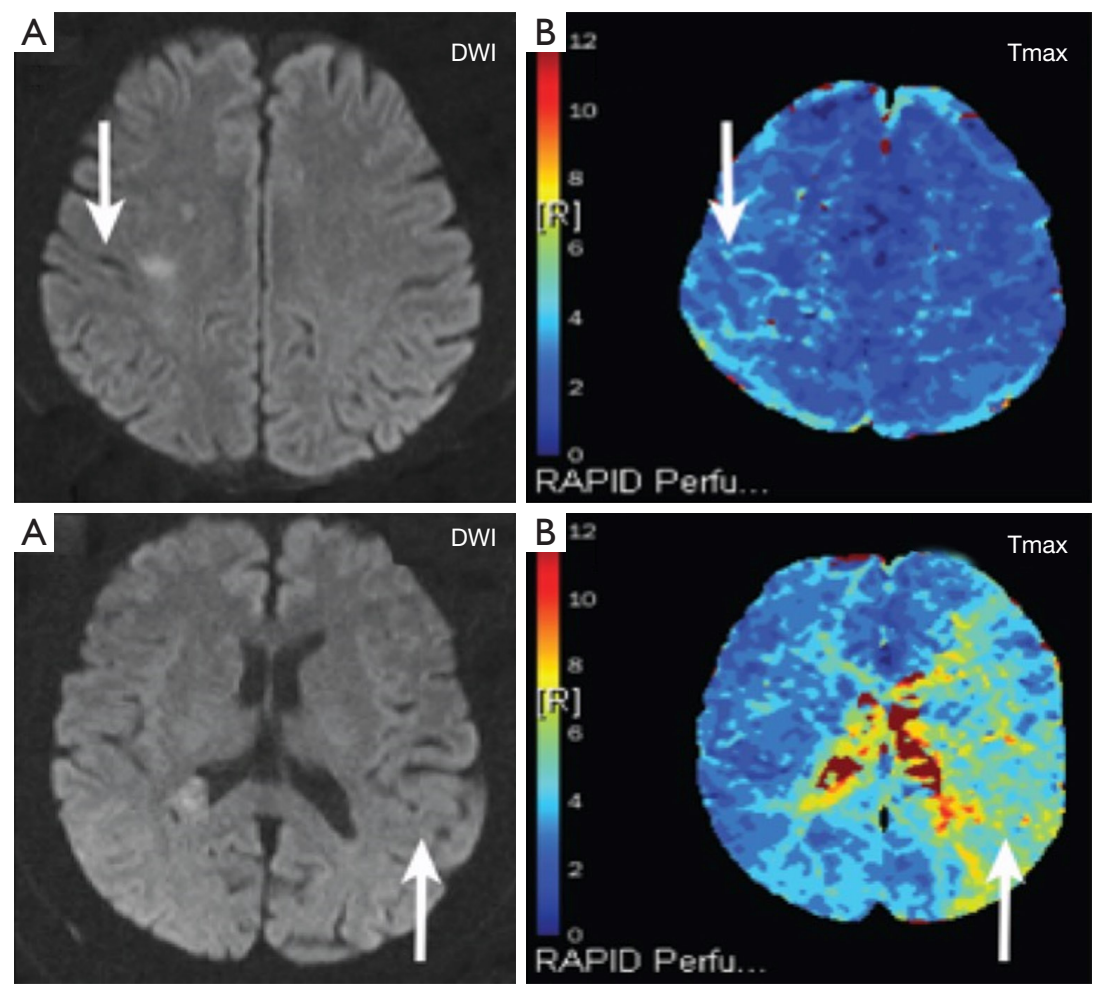

Figure 2 Diffusion and perfusion abnormalities of two TIA patients. (A,B) A 49-year-old male who presented with aphasia and decreased weakness in the left arm, MR scan was completed 13 days after TIA onset. The DWI lesion (A, arrow, areas of visual hyperintensity) was co-registered with the hypoperfusion area on the time to maximum of residue function (Tmax $\geq 4 \mathrm{~s}$ ) map (B, arrow, light blue areas). (C,D) an 83-year-old female who presented with aphasia, MR scan was completed 3 hours after TIA onset. The DWI map (C, arrow, no areas of visual hyperintensity) was normal and the same brain area showed low perfusion on the Tmax $\geq 4 \mathrm{~s}$ map (D, arrow, yellow and blue areas). TIA, transient ischemic attack; DWI, diffusion-weighted imaging. 
Table 3 Factors independently associated with $T \max >4 \mathrm{~s}>0 \mathrm{~mL}$ in hemispheric DWI-negative TIA patients

\begin{tabular}{lcccc}
\hline Risk factors & OR $(95 \% \mathrm{Cl})$ & P value & Adjusted OR $(95 \% \mathrm{Cl})$ & $\mathrm{P}$ value \\
\hline Perfusion_first & $0.90(0.81-0.99)$ & 0.038 & $0.898(0.808-0.999)$ & 0.048 \\
Male & $2.86(0.72-11.37)$ & 0.136 & $2.5(0.57-11.02)$ & 0.226 \\
\hline
\end{tabular}

$\mathrm{Cl}$, confidence interval; OR, odds ratio; Tmax $>4 \mathrm{~s}$, time to maximum of the residue function $\geq 4$ seconds; Perfusion_first, symptom onset to the first MR perfusion (days). DWI, diffusion-weighted imaging; TIA, transient ischemic attack.

Table 4 Factors independently associated with Tmax $>4 \mathrm{~s} \geq 10 \mathrm{~mL}$ in hemispheric DWI-negative TIA patients

\begin{tabular}{lcccc}
\hline Risk factors & OR $(95 \% \mathrm{Cl})$ & P value & Adjusted OR $(95 \% \mathrm{Cl})$ & $\mathrm{P}$ value \\
\hline Times_before & $0.73(0.48-1.10)$ & 0.13 & $0.68(0.44-1.03)$ \\
Perfusion_first & $0.92(0.84-1.02)$ & 0.12 & $0.94(0.84-1.05)$ \\
Weakness & $4.22(1.20-14.9)$ & 0.025 & $7.41(1.57-34.89)$ & 0.294 \\
\hline
\end{tabular}

$\mathrm{Cl}$, confidence interval; OR, odds ratio; Tmax $>4 \mathrm{~s}$, time to maximum of the residue function $\geq 4$ seconds. Times_before, times from onset to hospitalization; Perfusion_first, symptom onset to the first MR perfusion (days). DWI, diffusion-weighted imaging; TIA, transient ischemic attack.

and possible risk factors associated with MR diffusion abnormality. Comparisons of these variables between subjects with and without DWI lesions showed no significant difference in these clinical characteristics between the DWI-positive and DWI-negative groups. However, Male patients tended to develop DWI positive lesions after a TIA ( $\mathrm{P}=0.089$, OR 2.75, 95\% CI: 0.86-8.23).

\section{Prediction of MRI perfusion abnormality}

In the present study, we divided patients with hypoperfusion into two groups based on the volume of Tmax: Tmax > $4 \mathrm{~s}>0 \mathrm{~mL}$ and Tmax $>4 \mathrm{~s} \geq 10 \mathrm{~mL}$. The prevalence of MR perfusion Tmax $>4 \mathrm{~s}>0 \mathrm{~mL}$ and $T \max >4 \mathrm{~s} \geq 10 \mathrm{~mL}$ was $77.3 \%(51 / 66)$ and $50 \%(33 / 66)$, respectively.

\section{Prediction of Tmax $>4 s>0$ mL in hemispheric DWI- negative TIA patients}

In univariate binary logistic regression analysis, days from the first onset to perfusion (perfusion_first) was independently associated with MR perfusion lesions $(\mathrm{P}=0.038$, OR 0.90, 95\% CI: 0.81-0.99). Males ( $\mathrm{P}=0.14$, OR 2.86, 95\% CI: $0.72-11.37$ ) tended to be related to perfusion abnormalities after TIAs (Table 3).

Multivariate step regression modelling was performed for predictors with $\mathrm{P}$ values $<0.20$. The multivariate logistic regression analysis was performed to show the correlation between perfusion_first, male gender, and MR perfusion abnormalities (Table 3). The longer the time from onset to admission was, the longer the interval from the first onset to examination was, the less likely the patients were to develop hypoperfusion on PWI maps. The adjusted odds ratios were 0.9 (95\% CI: $0.808-0.999, \mathrm{P}=0.048$ ).

\section{Prediction of Tmax $>4 s \geq 10 \mathrm{~mL}$ in hemispheric DWI- negative TIA patients}

In univariate binary logistic regression analysis, limb weakness $(\mathrm{P}=0.025, \mathrm{OR}=4.22,95 \% \mathrm{CI}: 1.20-14.9)$ was independently associated with MR perfusion lesions. Times before $(\mathrm{P}=0.13, \mathrm{OR}=0.73,95 \%$ CI: $0.48-1.10)$ and perfusion_first (days from the first onset to perfusion) $(\mathrm{P}=0.12$, OR 0.92, 95\% CI: 0.84-1.02) tended to be related to perfusion abnormalities (Tmax $>4 \mathrm{~s} \geq 10 \mathrm{~mL}$ ) after TIAs.

Multivariate step regression modelling was performed for predictors with their $\mathrm{P}$ values $<0.20$. The multivariate logistic regression analysis was performed to show the correlation between perfusion_first, times_before, weakness and MR perfusion Tmax $>4 \mathrm{~s} \geq 10 \mathrm{~mL}$. We found that PWI lesions (Tmax $>4 \mathrm{~s} \geq 10 \mathrm{~mL}$ ) were associated with limb weakness (adjusted OR $=7.41,95 \%$ CI: 1.57-34.89, $\mathrm{P}=0.011)$ (Table 4).

\section{Discussion}

To the best of our knowledge, this is the first study that presented the risk factors for perfusion abnormalities using 
MRI Tmax in TIA patients with a compromised anterior circulation in a Chinese population. Male TIA patients tend to develop DWI positive lesions. In addition, limb weakness is an independent risk factor for $T \max >4 \mathrm{~s} \geq 10 \mathrm{~mL}$ hypoperfusion in TIA patients without DWI lesions.

\section{Prevalence and risk factors of anterior circulation TLAs with positive DWI}

The present study showed a $30.3 \%(20 / 66)$ prevalence of positive DWI lesions among anterior circulation TIA patients, which is similar to results of others including Stanford Stroke Center which showed a prevalence of $34.9 \%$ (15/43) (8), Yeungnam University Medical Center which showed a prevalence of $37.5 \%(24 / 64)$ (9), and consistent with a recent systematic review (28).

We found that DWI lesions in male patients tended to be correlated with the volume of Tmax $>4 \mathrm{~s}$.

The incidence of stroke in males is higher than that in females (29). Pre-clinical and clinical studies strongly suggest that sex plays a key role in stroke due to an amalgamation of factors including sex chromosomes, exposure to sex hormones, and the microenvironment of the brain and blood vessels $(29,30)$. Other factors previously known to be associated with DWI abnormalities, including symptom duration, speech impairment, motor weakness, atrial fibrillation, and ICA stenosis over $50 \%(8,28)$, were not confirmed in our study. A previous review showed that symptom duration over 60 minutes was associated with the presence of DWI lesions in TIA patients (28). The proportion of a TIA lasting 60 minutes or longer was in the range of $30 \%$ to $83 \%$, which was greater than that of a general population of patients with TIAs (28). Another study demonstrated that symptom duration greater than 12 hours was associated with presence of DWI lesions (8). However, in the present study, the median duration of TIAs was 20 minutes with an IQR of 5-68 minutes, which was much shorter than that of previous studies. Apart from this, there were only 5 patients with atrial fibrillation in the present study, and the proportion of patients with large vessel stenosis or with severe limb disorders was small. This might be due to the wide application of intravenous thrombolysis, thrombectomy, and multimodal imaging in China (31-35).

Multiple CT or MR perfusion sequences and analysis softwares are available to assess cerebral perfusion $(36,37)$. Previous studies used Time to Peak (TTP) and mean transit time (MTT) to detect persistent perfusion lesions following
TIAs $(8,24,38,39)$. The application of automated Tmax to identify ischemic penumbra has been widely used recently $(23,40)$. A previous study has shown that the automated Tmax map is more sensitive in assessing perfusion lesions in patients with TIA than TTP and MTT (24). We used an automated arterial input function detection and delayinvariant deconvolution software named RAPID to automatically evaluate Tmax maps (23). Tmax $>4$ s seems to be optimal for early assessment of critically hypoperfused brain areas, which is consistent with a previous report (25). The threshold Tmax $>4 \mathrm{~s}$ at a volume of $10 \mathrm{~mL}$ is optimal for predicting infarct growth with the maximal sensitivity and specificity (15). Hence, in the present study we considered Tmax $\geq 10 \mathrm{~mL}$ as an indicator of significantly low perfusion.

\section{Prevalence of MR perfusion abnormality}

Our study showed a 73.9\% (34/46) prevalence of MR perfusion abnormality (Tmax $>4 \mathrm{~s}>0 \mathrm{~mL}$ ) in patients with DWI-negative TIAs and 45.7\% (21/46) (Tmax >4 s $\geq 10 \mathrm{~mL}$ ) had an acute focal PWI lesion without showing a DWI lesion. This rate is similar to that of a Canadian study with a prevalence of $42 \%$ (15), but higher than that of the other two studies with a prevalence of $23 \%$ (16) and 25\% (9), respectively.

There are two main reasons for this high prevalence in the present study. Firstly, the difference between our and other studies is likely due to the use of discrete measurements of perfusion. Five different perfusion parameters are most commonly used to define perfusion abnormalities (37): time to peak contrast concentration (TTP) (9), cerebral blood volume (CBV), cerebral blood flow (CBF) (16), mean transit time (MTT) (9), and time at which the deconvolved residue function reaches its maximum value (Tmax) (16). However, automated Tmax is more sensitive than MTT in revealing hypoperfusion lesions in TIA patients with negative DWI findings $(9,24)$. Secondly, different analysis methods may produce different results. RAPID, an automated software, was used in the present study to calculate the volume of perfusion. This software might be more sensitive than Matlab 7.4 (The Mathworks) (15) or observers $(9,16)$. Therefore, in the present study, the proportion of perfusion abnormalities calculated by RAPID on Tmax maps is higher than previous studies.

\section{Risk factors associated with MR perfusion abnormalities}

There are multiple potential clinical risk factors of $M R$ 
perfusion abnormalities in the context of TIA. Previous studies investigated the relationship between the time of symptom onset, baseline PWI and perfusion abnormalities in patients with anterior circulation TIAs. It was found that there was an increased incidence of PWI abnormalities in patients scanned within 12 hours of symptom onset (8). In the present study, the median time of the first symptom onset to the baseline MR perfusion (perfusion_first) is 5 days with an IQR of 2-11 days. We found that the shorter the time from onset to perfusion is, the easier it is to find hypoperfusion after a TIA. This was further confirmed in the multiple logistic regression analysis, suggesting that time from symptom onset to the baseline PWI is an independent predictor of perfusion abnormalities in patients with hemispheric TIAs.

The primary finding of the present study was that limb weakness was an independent risk factor of hypoperfusion greater than $10 \mathrm{~mL}$. Previous findings suggest that acute PWI lesions may be due to persistent microvascular injury without reperfusion which results in hypoperfusion $(9,41)$. Lesions leading to limb weakness in the anterior circulation are mainly located in areas with much oxygen consumption, poor collateral circulation, and susceptibility to hypoxia and ischemia.

In the present study, the widely used $\mathrm{ABCD}^{2}$ score was not associated with perfusion deficit, which is similar to what was reported by previous studies $(16,42)$. The possible explanation for this might be that the $\mathrm{ABCD}^{2}$ score is based on patients' clinical factors and does not include information about brain hemodynamics though unilateral limb weakness is an important part of the $\mathrm{ABCD}^{2}$ scoring system. The present study found that limb weakness was a significant predictor of hypoperfusion over $10 \mathrm{~mL}$ and limb weakness may be useful in identifying high-risk TIA patients.

\section{MRA and perfusion}

Two studies have suggested that hypoperfusion lesions are less likely to be detected in TIA subjects who have a significant intracranial occlusion or carotid stenosis that can be visualized on MRA $(8,9)$. In the present study, $25.8 \%$ of the patients had evidence of an ipsilateral proximal artery stenosis or occlusion on MRA. However, acute PWI lesions were not associated with vascular stenosis. The median volume of Tmax $>4$ s was $9 \mathrm{~mL}$, which may reflect occlusion of distal intracranial vessels that were not well visualized on MRA. Therefore, MRA findings seem to be an inadequate surrogate for PWI abnormalities in TIA patients due to anterior circulation lesions.

The present study has a number of limitations. Firstly, it is a retrospective study that may introduce unknown subject selection bias. Moreover, it has a relatively small sample size that does not have enough power. Hence, prospective studies with a large sample size are required to confirm our findings. Secondly, it is a cross-sectional study and can not pinpoint the direct causality between hypoperfusion and the risk factors in TIA patients. A longitudinal design can help to investigate the direct causal relationship between risk factors and MR hypoperfusion in future studies. Thirdly, all subjects were recruited from one local hospital. Therefore, conclusions should be treated with caution. Fourthly, in the present study, imaging and clinical followup were not completed. It is unclear whether the perfusion abnormalities observed were reversible or have progressed to cerebral infarction after initial imaging. In addition, it was impossible to determine whether some of the PWI lesions were chronic.

In conclusion, hypoperfusion could be found in hemispheric TIA patients even when PWI was examined 48 hours after the symptom onset. The shorter the time from symptom onset to the initial PWI is, the more likely it is to find PWI lesions. Men with limb weakness are more likely to have severe hypoperfusion and may develop cerebral infarction. However, larger prospective studies are needed to confirm these findings.

\section{Acknowledgments}

We thank Dr Xi Zhang, Yangyang Huang, and Linglei Meng for their support in providing relevant cases.

Funding: The present study was Supported by the Fundamental Research Funds for the Central Universities awarded to Dr Yue Wang (NO. 22120180281) and a grant from Shanghai Health Bureau Science and Research Projects Foundation awarded to Dr Yu Luo (grant number 201740137).

\section{Footnote}

Conflicts of Interest: The authors have no conflicts of interest to declare.

Ethical Statement: The authors are accountable for all aspects of the work in ensuring that questions related to the accuracy or integrity of any part of the work are 
appropriately investigated and resolved. Ethical approval for this study was obtained from the Human Research Ethics Committee of Shanghai Fourth People's Hospital Affiliated to Tongji University School of Medicine. Written informed consent was obtained from all subjects.

\section{References}

1. Easton JD, Saver JL, Albers GW, et al. Definition and evaluation of transient ischemic attack: a scientific statement for healthcare professionals from the American Heart Association/American Stroke Association Stroke Council; Council on Cardiovascular Surgery and Anesthesia; Council on Cardiovascular Radiology and Intervention; Council on Cardiovascular Nursing; and the Interdisciplinary Council on Peripheral Vascular Disease. The American Academy of Neurology affirms the value of this statement as an educational tool for neurologists. Stroke 2009;40:2276-93.

2. Liu M, Wu B, Wang WZ, et al. Stroke in China: epidemiology, prevention, and management strategies. Lancet Neurol 2007;6:456-64.

3. Lavallee PC, Meseguer E, Abboud H, et al. A transient ischaemic attack clinic with round-the-clock access (SOSTIA): feasibility and effects. Lancet Neurol 2007;6:953-60.

4. Amarenco P, Lavallee PC, Labreuche J, et al. One-Year Risk of Stroke after Transient Ischemic Attack or Minor Stroke. N Engl J Med 2016;374:1533-42.

5. Kim AS, Easton JD, Johnston SC. Risk of Stroke after Transient Ischemic Attack or Minor Stroke. N Engl J Med 2016;375:386-7.

6. Castle J, Mlynash M, Lee K, et al. Agreement regarding diagnosis of transient ischemic attack fairly low among stroke-trained neurologists. Stroke 2010;41:1367-70.

7. Cereda CW, George PM, Inoue M, et al. Inter-rater agreement analysis of the Precise Diagnostic Score for suspected transient ischemic attack. Int J Stroke 2016;11:85-92.

8. Mlynash M, Olivot JM, Tong DC, et al. Yield of combined perfusion and diffusion MR imaging in hemispheric TIA. Neurology 2009;72:1127-33.

9. Lee J, Inoue M, Mlynash M, et al. MR perfusion lesions after TIA or minor stroke are associated with new infarction at 7 days. Neurology 2017;88:2254-9.

10. Brazzelli M, Chappell FM, Miranda H, et al. Diffusionweighted imaging and diagnosis of transient ischemic attack. Ann Neurol 2014;75:67-76.

11. van Rooij FG, Vermeer SE, Goraj BM, et al. Diffusion- weighted imaging in transient neurological attacks. Ann Neurol 2015;78:1005-10.

12. Hotter B, Galinovic I, Kunze C, et al. High-resolution diffusion-weighted imaging identifies ischemic lesions in a majority of transient ischemic attack patients. Ann Neurol 2019;86:452-7.

13. Hurford R, Li L, Lovett N, et al. Prognostic value of "tissue-based" definitions of TIA and minor stroke: Population-based study. Neurology 2019;92:e2455-61.

14. Kleinman JT, Greg Z, Michael M, et al. Automated perfusion imaging for the evaluation of transient ischemic attack. Stroke 2012;43:1556-60.

15. Asdaghi N, Hill MD, Coulter JI, et al. Perfusion MR predicts outcome in high-risk transient ischemic attack/ minor stroke: a derivation-validation study. Stroke 2013;44:2486-92.

16. Grams RW, Kidwell CS, Doshi AH, et al. Tissue-Negative Transient Ischemic Attack: Is There a Role for Perfusion MRI? AJR Am J Roentgenol 2016;207:157-62.

17. Asdaghi N, Hameed B, Saini M, et al. Acute perfusion and diffusion abnormalities predict early new MRI lesions 1 week after minor stroke and transient ischemic attack. Stroke 2011;42:2191-5.

18. Olivot JM, Mlynash M, Thijs VN, et al. Relationships between infarct growth, clinical outcome, and early recanalization in diffusion and perfusion imaging for understanding stroke evolution (DEFUSE). Stroke 2008;39:2257-63.

19. Ostergaard L, Weisskoff RM, Chesler DA, et al. High resolution measurement of cerebral blood flow using intravascular tracer bolus passages. Part I: Mathematical approach and statistical analysis. Magn Reson Med 1996;36:715-25.

20. Ostergaard L. Cerebral perfusion imaging by bolus tracking. Top Magn Reson Imaging 2004;15:3-9.

21. Rao V, Christensen S, Yennu A, et al. Ischemic Core and Hypoperfusion Volumes Correlate With Infarct Size 24 Hours After Randomization in DEFUSE 3. Stroke 2019;50:626-31.

22. Albers GW, Marks MP, Kemp S, et al. Thrombectomy for Stroke at 6 to 16 Hours with Selection by Perfusion Imaging. N Engl J Med 2018;378:708-18.

23. Straka M, Albers GW, Bammer R. Real-time diffusionperfusion mismatch analysis in acute stroke. J Magn Reson Imaging 2010;32:1024-37.

24. Kleinman JT, Zaharchuk G, Mlynash M, et al. Automated perfusion imaging for the evaluation of transient ischemic attack. Stroke 2012;43:1556-60. 


\section{Page 10 of 10}

25. Olivot JM, Mlynash M, Thijs VN, et al. Optimal Tmax threshold for predicting penumbral tissue in acute stroke. Stroke 2009;40:469-75.

26. Lansberg MG, Lee J, Christensen S, et al. RAPID automated patient selection for reperfusion therapy: a pooled analysis of the Echoplanar Imaging Thrombolytic Evaluation Trial (EPITHET) and the Diffusion and Perfusion Imaging Evaluation for Understanding Stroke Evolution (DEFUSE) Study. Stroke 2011;42:1608-14.

27. Mlynash M, Eyngorn I, Bammer R, et al. Automated method for generating the arterial input function on perfusion-weighted MR imaging: validation in patients with stroke. AJNR Am J Neuroradiol 2005;26:1479-86.

28. Redgrave JN, Coutts SB, Schulz UG, et al. Systematic review of associations between the presence of acute ischemic lesions on diffusion-weighted imaging and clinical predictors of early stroke risk after transient ischemic attack. Stroke 2007;38:1482-8.

29. Poorthuis MH, Algra AM, Algra A, et al. Female- and Male-Specific Risk Factors for Stroke: A Systematic Review and Meta-analysis. JAMA Neurol 2017;74:75-81.

30. Chauhan A, Moser H, McCullough LD. Sex differences in ischaemic stroke: potential cellular mechanisms. Clin Sci (Lond) 2017;131:533-52.

31. Bivard A, Parsons M. Tissue is more important than time: insights into acute ischemic stroke from modern brain imaging. Curr Opin Neurol 2018;31:23-7.

32. Wang Y, Li Z, Zhao X, et al. Stroke care quality in China: Substantial improvement, and a huge challenge and opportunity. Int J Stroke 2017;12:229-35.

33. Kawano H, Bivard A, Lin L, et al. Perfusion computed

Cite this article as: Wang Y, Xiao J, Luo Y, Wang S, Liang H, Jin L. Risk factors of perfusion and diffusion abnormalities on MRI in hemispheric TIA: a case-control study. Ann Transl Med 2019;7(24):808. doi: 10.21037/atm.2019.12.69
Wang et al. Limb weakness predicts perfusion abnormalities

tomography in patients with stroke thrombolysis. Brain 2017;140:684-91.

34. Bivard A, Levi C, Lin L, et al. Validating a Predictive Model of Acute Advanced Imaging Biomarkers in Ischemic Stroke. Stroke 2017;48:645-50.

35. Chen C, Parsons MW, Clapham M, et al. Influence of Penumbral Reperfusion on Clinical Outcome Depends on Baseline Ischemic Core Volume. Stroke 2017;48:2739-45.

36. Liu L, Ding J, Leng X, et al. Guidelines for evaluation and management of cerebral collateral circulation in ischaemic stroke 2017. Stroke Vasc Neurol 2018;3:117-30.

37. Copen WA, Schaefer PW, Wu O. MR perfusion imaging in acute ischemic stroke. Neuroimaging Clin N Am 2011;21:259-83, $\mathrm{x}$.

38. Restrepo L, Jacobs MA, Barker PB, et al. Assessment of transient ischemic attack with diffusion- and perfusion-weighted imaging. AJNR Am J Neuroradiol 2004;25:1645-52.

39. Krol AL, Coutts SB, Simon JE, et al. Perfusion MRI abnormalities in speech or motor transient ischemic attack patients. Stroke 2005;36:2487-9.

40. Nogueira RG, Jadhav AP, Haussen DC, et al.

Thrombectomy 6 to 24 Hours after Stroke with a Mismatch between Deficit and Infarct. N Engl J Med 2018;378:11-21.

41. del Zoppo GJ, Mabuchi T. Cerebral microvessel responses to focal ischemia. J Cereb Blood Flow Metab 2003;23:879-94.

42. Nah HW, Kwon SU, Kang DW, et al. Diagnostic and prognostic value of multimodal MRI in transient ischemic attack. Int J Stroke 2014;9:895-901. 